\title{
Towards developing lessons learned and experience based factory in software development
}

\begin{abstract}
Software development is a highly knowledge intensive activity. During the software life cycle, knowledge and experiences are accumulated over time. One of the main knowledge sources in software development is lessons learned. Even though lessons learned have been a common practice for process improvement, however, there are arguments that lessons learned are not used effectively and organizations continuously fail to learn from past projects. In this paper, we propose utilizing lessons learned by transforming it into an experience base incorporated with software engineering life cycle for the purpose of sharing and future reuse. An initial model is formulated based on literature analysis and a preliminary study. The goal of the study is to assess the experts' perception on the model formulation in terms of its importance in each software development phase, and which knowledge element is more valuable as future reuse. Additionally, results from the study show that such model can bring positive impact to individuals as well as organizations.
\end{abstract}

Keyword: Knowledge management; Software development; Experience factory; Lessons learned 